\title{
Editorial: Special Issue on Ionic Fluids and Its Biological Application
}

\author{
Xiaolin $\mathrm{CHENG}^{1}$, Zhenli XU ${ }^{2}$ \\ ${ }^{1}$ (Oak Ridge National Lab, Oak Ridge, TN 37831, USA, chengx@ornl.gov) \\ ${ }^{2}$ (Shanghai Jiao Tong University, Shanghai 200240, China, xuzl@sjtu.edu.cn)
}

Ionic fluids have ever been of central interest in physical chemistry, but in recent years have also permeated in biology and resulted in numerous biological applications. It has been well recognized that life occurs in ionic fluids, thus the properties and behavior of ionic fluids are critical to understand many biological phenomena. There have been tremendous progresses during the past a few decades in the theories of ionic fluids, from the cluster expansion theory of McMillan-Mayer, integral equation approaches with the hypernetted chain (HNC)/mean spherical approximation (MSA) and Percus-Yevick (PY) equations, classical density functional theory to recent variational field theory that includes both structure and flow. Theoretical studies have begun to consider more realistic interactions between various components in ionic fluids. However, two properties render ionic fluid theories difficult, namely the long-range correlation of Coulomb interactions and the size/shape effect of various components in ionic fluids. These interactions have distinct consequences on the structure of ionic fluids. Additionally, the long-range nature of the Coulomb interactions not only causes technical problems in theory, but also challenges numerical simulations.

It is impossible to include all aspects of ionic fluid theories and applications in one special issue. We hope this Special Issue of Interdisciplinary Sciences: Computational Life Sciences provides a snapshot of recent developments in ionic fluid theories and computational studies of biological systems with ionic fluid theories and simulations which is dedicated to Prof. Lesser Blum who is one of the pioneers in shaping these fields, especially, the MSA. An overview of the papers is as follows.

1. Montes-Pérez et al. present the mean spherical approximation (MSA) for a fluid with an effective potential that contains the hard sphere plus one Yukawa (HS1Y) tail. Starting from Blum-Høye solution for OrnsteinZernike (OZ) equation of the system, they obtain the thermodynamics and static structure factor in terms of a scaling parameter.

2. Gazzillo and Giacometti review application of integral equation theory to complex fluids, with a particular emphasis on the effects of polydispersity and anisotropy on their structural and thermodynamic properties. They discuss applications to colloidal mixtures, globular proteins and patchy colloids, within a unified framework.

3. The general solution to the nonlinear Poisson-Boltzmann equation for two parallel charged plates, either inside a symmetric electrolyte, or inside a 2:1 asymmetric electrolyte, is found in terms of Weierstrass elliptic functions. From this, Xing derives some exact asymptotic results for the interaction between charged plates, as well as the exact form of the renormalized surface charge density.

4. Maruyama et al. review their recent studies on the molecular recognition and stability of biomolecules in aqueous solutions, based on the statistical mechanics of molecular liquids, or the 3D-RISM/RISM theory. A special stress is put on roles of electrolytes in determining the stability of biomolecules.

5. Vericat et al. calculate the pair distribution function of the electron gas using a parameterized generalization of quantum hypernetted chain approximation, in which the parameters are obtained by optimizing the system energy with a genetic algorithm.

6. Xu proposes a new method for fast calculation of the electric field of spaced spheres using the multiple reflection expansion. The method uses a technique of recursive reflections among the spherical interfaces based 
on a formula of the multiple image representation, resulting in a simple, accurate and close-form expression for the surface polarization charges.

7. Rodriguez and Garcia study concentration dependence of $\mathrm{NaCl}$ ion distributions around Dipalmitoylphosphatidylcholine (DPPC) lipid bilayers using molecular dynamics simulations. They find that most Na ions are bound to the lipid carbonyls, while $\mathrm{Cl}$ ions form an ionic cloud around the lipid choline groups for low number of ions per lipids (1:16 and 1:8). The residence time of $\mathrm{Na}$ ions bound to the lipid is longer than 40 $\mathrm{ns}$, while $\mathrm{Cl}$ ions exchange faster than the nanoseconds timescale.

8. Mostofian et al. perform molecular dynamics simulations of a cellulose microfibril in the ionic liquid 1-butyl3-methylimidazolium chloride $(\mathrm{BmimCl})$ and compare the solute structure and the solute-solvent interactions at the interface with those from corresponding simulations in water. The results indicate that hydrophilic surfaces are a preferred site of interaction between cellulose and the ionic liquid. Furthermore, the glucose units with these solvent-oriented hydroxymethyls are surrounded by the heterocyclic organic cation in a preferred parallel orientation, suggesting a direct and distinct interaction scheme between cellulose and $\mathrm{BmimCl}$.

In summary, the eight papers represent some of the latest and most promising research results in this traditional but continually evolving field. These advances will make ionic fluid theories possible for a rapidly increasing number of biological systems and further establish them as an integral tool for the understanding of biological phenomena. We are confident that this special issue will stimulate further research in this area. 